\title{
La regeneración natural del bosque en tierras abandonadas como estrategia de restauración ${ }^{1}$
}

Robin L. Chazdon², David Lindenmayer ${ }^{3}$, Renato Crouzeilles ${ }^{4}$, José María Rey Benayas 5 , Elena Lazos Chavero ${ }^{6}$, Manuel R. Guariguata ${ }^{7}$

\section{Mensajes principales}

- En condiciones apropiadas, las tierras que fueron sometidas al uso agrícola o pastoril pueden revertir a bosques mediante el proceso de regeneración natural ya sea de forma asistida o sin asistencia. Estos nuevos bosques conservan biodiversidad, brindan un amplio espectro de bienes y servicios ecosistémicos, y apoyan economías y medios de vida rurales.

- La planificación integral de uso del suelo y la aplicación de enfoques de priorización espacial pueden contribuir a asegurar que los bosques regenerados naturalmente persistan en el tiempo sin poner en riesgo la producción de alimentos, recreación, o la cosecha de madera. Sin embargo, las políticas y mecanismos para promover soluciones holísticas, se encuentran poco desarrolladas, sobre todo en el trópico.

- De igual forma se necesitan políticas innovadoras, a múltiples niveles de gobernanza, para alcanzar así la escala necesaria que permita aprovechar la acción de la naturaleza en forma costo-efectiva y restaurar los bosques nativos.

Cuando los campos de cultivo y las pasturas que en el pasado albergaron bosque caen en desuso, un proceso natural de regeneración —-también denominado sucesión secundaria, restauración espontánea o restauración pasiva - con frecuencia deriva en el desarrollo de un nuevo ecosistema boscoso que recupera gradualmente muchas de sus propiedades originales. En este proceso, la vegetación se regenera de muchas maneras: por la liberación de semillas en respuesta a las quemas, a partir de semillas en el suelo o de semillas depositadas recientemente por el viento o animales, gracias al rebrote de tocones, o por propágulos vegetativos. Un bosque que atraviesa un proceso de regeneración natural luego del uso agrícola es un sistema socioecológico en transición (sensu Lambin y Meyfroidt, 2010), más allá de la simple dicotomía "bosque" vs. "no bosque".
En anticipación a la Década de las Naciones Unidas para la Restauración de los Ecosistemas 2021-2030, es oportuno considerar dónde y cómo la regeneración natural de bosques en tierras previamente utilizadas para cultivos o pastoreo, puede contribuir a los esfuerzos internacionales en la actualidad para restaurar los ecosistemas forestales degradados (Chazdon y Brancalion, 2019). Bastin et al. (2019) calculan que 9 millones de $\mathrm{km}^{2}$ a nivel global son idóneos para reforestar (incluyendo bosques regenerados naturalmente). De hecho, y por múltiples razones, grandes áreas ya se están regenerando naturalmente en varias regiones del mundo (Figura 1). Por ello, es tiempo de reconocer el valor de los bosques regenerados de manera natural y de insertar con firmeza este cambio en el uso de la tierra dentro del contexto de las políticas ambientales hacia la creación de paisajes multifuncionales que sustenten a las personas y el ambiente. Esto, porque la regeneración natural del bosque que ocurre luego del abandono de la

\footnotetext{
1 Traducido y modificado del artículo Fostering natural forest regeneration on former agricultural land through economic and policy interventions por Chazdon RL, Lindenmayer D, Guariguata MR, Crouzeilles R, Rey Benayas J, Lazos-Chavero E (2020), en Environmental Research Letters https://doi.org/10.1088/1748-9326/ab79e6

2 Universidad de la Costa del Sol, Australia
}

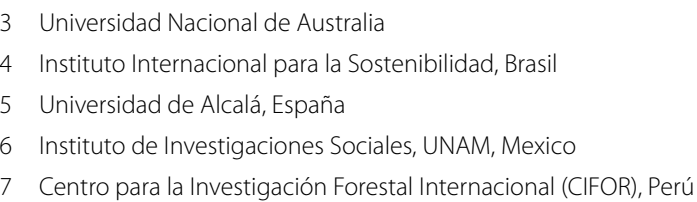


Puerto Rico - Ganancia neta de $311,5 \mathrm{~km}^{2}$ de $2000-2010$ (Wang et al 2017)

Córcega, Francia - Ganancia neta de bosques de Quercus ilex (+ 3,40 \% año-1 entre 1975 y 2000) (San Roman Sanz et al 2013)
Europa - Con base en información disponible del año 2010, 133 M ha, o cerca de $68 \%$ de los bosques se regeneraron naturalmente (FAO 2015)

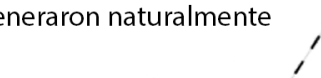

América Latina y El Caribe - Ganancia neta de $2209930 \mathrm{~km}^{2}$ de 2001 a 2014 (Nanni et al 2019)

Antillas Mayores, Caribe Ganancia neta de $801 \mathrm{~km}^{2}$ desde 2001-2010. Casi toda la ganancia $(99,8 \%)$ ocurrió en Cuba, pero también se incrementó vegetación leñosa en Puerto Rico y la República Dominicana (Álvarez-Berríos et al 2013)

Guanacaste, Costa Rica - Ganancia neta de cobertura forestal ele $23,4 \%$ desde 1986-2005 (Calvo-Alvarado et al 2009)

Amazonía brasileña - Ganancia quintuplicada desde 1980-2012, de < 3 millones ha hasta , más de 15 millones ha (Jakovac et al 2016 \& Aguiar et al 2016) Montañas de los Andes tropicales del suelo en los Andes entre 2001 y 2014 conllevó a una ganancia neta de $\sim 0.5 \mathrm{M}$ ha de vegetación leñosa (Aide et al 2019)

Estado de Mato Grosso, Brasil Desde 2008, 19,2\% de áreas previamente deforestadas experimentaron regeneración natural (Almeida et al 2016)

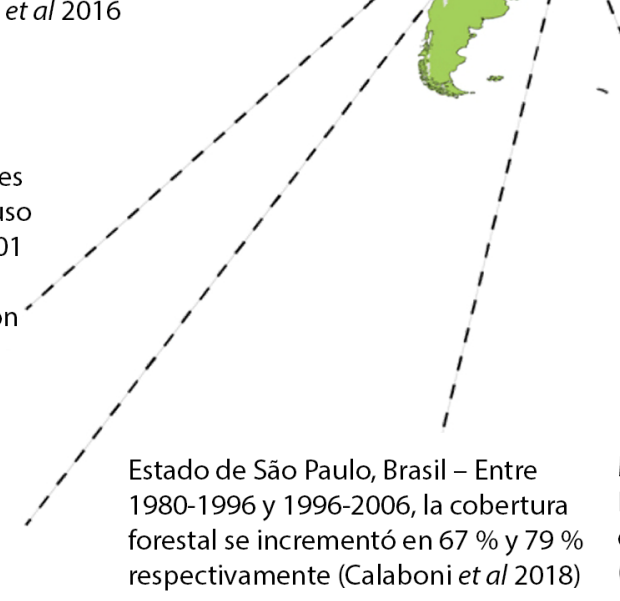

Alpes europeos -

Ganancia neta de 4,3\% por década desde 1990 forestal de $15 \%$ de 1982-2016 (Bebi et al 2017)

Suroeste - Sureste de Europa - En el suroeste y el sureste de Europa hubo una tendencia creciente en regeneración natural a lo largo de 20 años (FAO 2015)

Oeste de Ukrania - Ganancia neta de 2100 ha/año

Europa Oriental (20 países) - 6,4 M ha de regeneración natural en tierras agrícolas abandonadas desde 1985 hasta 2012 (Potapov et al 2015)

Figura 1. Casos documentados de regeneración forestal natural a gran escala en paisajes agrícolas en regiones templada, tropical y subtropical del mundo. En todos los casos, solo se reporta la regeneración natural y las ganancias en la cobertura de plantaciones forestales ha sido excluida. Los biomas de bosque tropical y subtropical se indican en color verde oscuro, en tanto que los biomas de bosque templado y boreal se indican en verde claro. 
actividad agrícola se produce en paisajes de uso múltiple, lo cual requiere atención hacia una amplia gama de problemas sociales y ecológicos, como lo resalta la reciente evaluación mundial del IPBES (Díaz et al, 2019). A su vez, el valor de los bosques regenerados naturalmente se ve fortalecido por el hecho de que, en recientes metaanálisis, se encontró que a largo plazo, los niveles de recuperación de la biodiversidad, la estructura del bosque y los valores de varios indicadores de funciones ecosistémicas son similares (o mayores) en bosques restaurados naturalmente en comparación con las plantaciones forestales (Crouzeilles et al, 2017). Dado el ímpetu internacional para impulsar la restauración forestal a gran escala, este documento busca ofrecer un punto de partida para ayudar a desarrollar enfoques que contemplen la regeneración natural del bosque a largo plazo.

\section{La importancia ambiental y socioeconómica de los bosques regenerados naturalmente}

Múltiples estudios sobre la regeneración natural de los bosques evidencian una recuperación gradual de las especies nativas (al compararse con los bosques de referencia), aunque la recuperación de la composición de especies puede tomar siglos en comparación con el número (riqueza) de especies, lo cual se alcanza en pocas décadas (Rozendaal et al, 2019). En las regiones tropicales, la recuperación de la biodiversidad y de la estructura del bosque puede ser de entre 34 a $56 \%$ y de 19 a $56 \%$ más alto, respectivamente, en bosques regenerados de forma natural en comparación con bosques plantados (Crouzeilles et al, 2017). Un factor importante al predecir la recuperación del bosque original es el grado de cobertura forestal a escala de paisaje. A nivel global, paisajes con más de $27 \%$ de cobertura forestal mostraron una tendencia consistente en la recuperación de biodiversidad. En conjunto, estos paisajes totalizan unos 238 millones de ha, lo que equivale a un $38 \%$ del área tropical y templada (Crouzeilles et al, 2019). De hecho, la fragmentación del bosque podría reducirse ( $y$, por ende, la conectividad aumentar) hasta un $44 \%$ en la mata atlántica de Brasil, si se permitiera que los $210000 \mathrm{~km}^{2}$ de tierra clasificada con alta capacidad de regeneración natural y asistida en este bioma se recuperen (Crouzeilles et al, 2020).

Pugh et al. (2019) calcularon que los bosques en regeneración natural (< 140 años) constituyen el 61,5\% de los 42,8 millones de $\mathrm{km}^{2}$ de áreas de bosques que existían en todo el mundo en 2010. Desde el año 2001 hasta el año 2010, el sumidero de carbono de estos bosques (1,3 Pg C año-1) constituyó el 60,5 \% del sumidero total de carbono. Los sumideros de carbono en bosques en regeneración se localizan principalmente en bosques caducifolios latifoliados y coníferas perennes en zonas templadas. Se estima que había un total de 2,9 millones de $\mathrm{km}^{2}$ de bosques en regeneración (< 100 años) en la bajura de la región neotropical, comparado con 4,0 millones de $\mathrm{km}^{2}$ de bosque primario en 2008. Si su crecimiento continuara por 40 años, estos bosques bajo regeneración natural podrían acumular una cantidad total estimada de carbono sobre el suelo de 8,48 Pg C.
Existe evidencia de que la regeneración natural mejora la retención de sedimentos y reduce la escorrentía superficial si se compara con las plantaciones forestales (Yang et al, 2018). Mediante la regeneración natural también pueden restaurarse los flujos hídricos en cuencas, al incrementarse la infiltración de lluvia en los suministros de agua edáfica (Filoso et al, 2017), aunque los efectos de los usos previos de la tierra y los enfoques de reforestación para recuperar la infiltración edáfica son complejos y poco estudiados (Lozano-Baez et al, 2019). No obstante estos beneficios, en algunos casos, el bosque que se regenera luego del abandono de un campo agrícola podría reducir la diversidad del paisaje y del hábitat, junto a otros efectos negativos sobre la biodiversidad (Queiroz et al, 2014), alteración de los flujos de agua (Bonnesoeur et al, 2019), e incluso la pérdida de paisajes culturales y técnicas tradicionales de manejo de la tierra cuando las tasas de migración humana son altas (Lasanta et al, 2017). La regeneración natural también puede conllevar al incremento de poblaciones animales que afectan negativamente la productividad agrícola y la salud de las personas (Byg et al, 2017). Todo esto subraya la necesidad de asegurar la amplia participación de las partes interesadas en las decisiones relacionadas con la gestión a escala de paisaje que incluyan bosques regenerados naturalmente.

La regeneración natural puede brindar beneficios económicos tanto directos como indirectos a los pobladores del bosque y las comunidades locales. Con políticas de apoyo y desarrollo de mercado, la regeneración natural puede mejorar, diversificar e incrementar la productividad a largo plazo de los sistemas agrícolas (Peltier et al, 2014), incluyendo los sistemas silvopastoriles (Kremen y Merenlender, 2018). Los beneficios económicos de la regeneración natural pueden compensar los costos de oportunidad del uso agrícola previo en estas tierras. Por ejemplo, en el estado de Sao Paulo, Brasil, se calculó que zonas bajo regeneración natural ahorrarían USD 1,17 millones anuales en costos de dragado de sedimentos del río (Strassburg et al, 2016). Una ventaja importante de la regeneración natural como herramienta de restauración es la reducción sustancial de los costos de implementación y mantenimiento comparado con plantaciones forestales (Cruz-Alonso et al, 2019), lo que abre paso a oportunidades de restauración de áreas considerablemente grandes (Chazdon y Guariguata, 2016). A lo largo del bioma de mata atlántica en Brasil, la restauración por medio de regeneración natural asistida podría disminuir los costos de implementación en USD 90,6 millones (un $77 \%$ ) en comparación con el establecimiento de plantaciones forestales (Crouzeilles et al, 2020).

\section{Dónde y por qué están creciendo nuevamente los bosques}

Los análisis de imágenes satelitales secuenciales y estudios en el terreno revelan que en muchas áreas alrededor del mundo los bosques tropicales y subtropicales se están regenerando naturalmente después del uso agrícola (Figura 1). Un análisis global de imágenes satelitales capturadas entre 1982 y 2016 mostró que la cobertura forestal está cambiando de manera dramática, con un aumento atribuido tanto a la regeneración natural como al establecimiento de plantaciones forestales (Song et al, 2018). En particular, un aumento 
de $15 \%$ en la cobertura forestal en el este de los Estados Unidos fue atribuido a la regeneración natural (Song et al, 2018). El mayor aumento se observó en Europa Oriental (35 \%), incluyendo a la Rusia europea y los bosques montanos de los Cárpatos (Song et al, 2018). En Europa Oriental, se atribuyó el aumento en la cobertura forestal a la regeneración natural en tierras agrícolas abandonadas luego del colapso de la Unión Soviética (Rudel et al, 2016). Sin embargo, a pesar de los muchos avances técnicos en la teledetección del bosque, no se cuenta con una evaluación precisa y sistemática de la localización de cobertura vegetal bajo regeneración natural en todo el mundo, debido principalmente a las dificultades para diferenciar entre las áreas de bosque nativo y las plantaciones forestales, además de las altas tasas de deforestación que sufren muchos bosques en regeneración (Reid et al, 2019). En comparación con las zonas subtropicales y templadas, la regeneración natural en los trópicos tiende a ser un fenómeno más reciente. Los bosques secundarios en el trópico son más jóvenes (media de 18 años) en comparación con los bosques caducifolios de la región templada (media de 52 años) y los bosques perennes de coníferas (media de 72 años) (Pugh et al, 2019).

En el siglo pasado, el abandono de la actividad agrícola en regiones montañosas de Europa conllevó tanto a la expansión de plantaciones forestales como de la regeneración natural, acompañado por la emigración de las zonas rurales a las ciudades y la intensificación de la agricultura en regiones de bajura (Cruz-Alonso et al, 2019). En varias regiones de África, la regeneración natural gestionada por los agricultores ha ocurrido en antiguas tierras de cultivo y en tierras de pastoreo (Smale et al, 2018). Bajo este enfoque se calcula que, solo en la República de Níger, se han transformado 70000 km² de tierras desnudas en zonas verdes de agroforestería productiva (Smale et al, 2018). En América Latina y el Caribe, Nanni et al. (2019) identificaron entre 2001 y 2014, 15 regiones que presentaron aumento de 2,2 millones de $\mathrm{km}^{2}$ de cobertura boscosa por regeneración natural. Este fenómeno se agrupó en cinco conglomerados que reflejan la topografía, grado de degradación agroecológica, cambio del clima, disminución de la población rural e incremento en la urbanización como variables predictivas de tal aumento de cobertura.

\section{Impulsores clave de la regeneración natural del bosque}

La regeneración natural del bosque refleja una gran cantidad de impulsores. Los factores ambientales que pueden influir en este proceso incluyen la calidad del suelo, la presencia de malezas o de especies invasoras que detienen el proceso de sucesión, o la dispersión inadecuada de semillas que restringe la colonización de especies nativas (Rey Benayas et al, 2008). La pérdida de primates y aves influye negativamente en la capacidad de regeneración natural del bosque (Gardner et al, 2019). En algunos casos, se necesitarán intervenciones para controlar las malezas, enriquecer la regeneración natural con especies de interés y mejorar la dispersión de semillas. La regeneración natural también puede ser asistida mediante el control o la eliminación del pastoreo de ganado y la prevención de incendios forestales (Gardner et al, 2019).
Muchos de los factores determinantes en el proceso de regeneración natural han sido investigados en una variedad de contextos y brindan una base para la planificación del uso del suelo. Planificar la ubicación de áreas bajo regeneración natural en relación con otras partes de las fincas (tales como potreros de pastoreo, cursos de agua y afloramientos rocosos) es fundamental para integrar de manera efectiva la producción agrícola con otros usos (Lindenmayer et al, 2016). En paisajes agrícolas, es más probable encontrar parches de regeneración forestal adyacentes a los remanentes de bosques primarios (Sloan et al, 2016); y cuando existe mayor cobertura forestal es más probable que proceda la regeneración natural y con mejores resultados en cuanto a biodiversidad (Crouzeilles et al, 2020). Una revisión sistemática sobre los impulsores de la expansión de la cobertura forestal en el trópico mediante regeneración natural descubrió que la proximidad a los remanentes del bosque, las pendientes empinadas, la alta cobertura forestal a escala de paisaje y la proximidad a los cursos de agua fueron los factores biofísicos más importantes (Borda-Niño et al, 2020).

En Mesoamérica y América del Sur, el abandono de la actividad agrícola está asociado a las expectativas por la mejora de oportunidades económicas representadas en los empleos en las ciudades cercanas, las operaciones de ecoturismo o las zonas industriales; y a menudo se acompaña de emigración de las zonas rurales (Hecht et al, 2015). En Nepal, donde los niveles de emigración fuera del país son altos, se observan tendencias similares (Oldekop et al, 2018). La emigración a otros países en Nepal se asoció al abandono de tierras agrícolas y generó la subsecuente regeneración natural del bosque (Oldekop et al, 2018). Los costos sociales y culturales de la emigración rural pueden ser altos, y hasta incluir la explotación y el aumento de la pobreza (García-Barrios et al, 2009). En algunas áreas, el ingreso de remesas que sigue a la emigración puede compensar parcialmente las pérdidas de mano de obra, manteniendo así algunas actividades agrícolas tradicionales en estas áreas (Oldekop et al, 2018).

\section{Barreras económicas y políticas para la regeneración natural del bosque}

Además de restricciones biofísicas, el proceso de regeneración natural del bosque enfrenta barreras socioeconómicas y jurisdiccionales. Un aspecto importante es la "invisibilidad" de la regeneración natural en el contexto de la restauración forestal, por al menos seis razones. Primero, las iniciativas de restauración a gran escala a menudo se conciben solo a través de plantar árboles (Biggs, 2018). En segundo lugar, los agricultores ven las primeras etapas de la regeneración natural como indeseables y desordenadas, o como un signo de mala gestión del suelo (Zahawi et al, 2014). Tercero, se dispone de conocimiento limitado para orientar las políticas y acciones que guíen hacia dónde podría ocurrir la regeneración natural, para calcular el área que se podría regenerar y cuánto tiempo llevaría obtener resultados sociales y ambientales específicos (Uriarte y Chazdon, 2016). Cuarto, para evaluar su efectividad socioeconómica hacen falta proyecciones económicas sólidas y modelos de negocio basados en la regeneración natural (Ding et al, 
2017). Quinto, la regeneración natural no se considera como una actividad que requiere intervención humana y, por lo tanto, no se puede hacer cumplir como política. Además, en algunos países, las leyes de reforma agraria obligan a los agricultores a cultivar las tierras, y las autoridades estatales pueden confiscar tierras no cultivadas o declarar tierras en barbecho como "tierras no utilizadas" o "degradadas" para ser usadas con otros fines (Duangjai et al, 2015).

Sin duda, una barrera importante es que la regeneración natural del bosque en áreas consideradas adecuadas para la agricultura genera altos costos de oportunidad y requiere que los propietarios reciban una compensación financiera adecuada si deciden transformar sus tierras agrícolas en bosque. En Costa Rica, los pagos a propietarios de tierras son de USD 125/ha/año por 16 años para la plantación de especies de árboles nativos, pero de solo USD 39/ha/año por 5 años para la protección de la regeneración natural (Porras y ChacónCascante, 2018). Dada la opción, los propietarios prefieren talar los bosques secundarios para establecer plantaciones de árboles o para cultivar productos básicos en lugar de regenerar bosques nativos (Shaver et al, 2015). En la Amazonía peruana, las etapas jóvenes de la regeneración natural son muy vulnerables a un segundo desmonte (Schwartz et al, 2017). En la Amazonía brasileña, 42040 km² de bosques secundarios producto de regeneración natural en pasturas abandonadas fueron convertidos en otro tipo de cobertura de suelo entre 2010 y 2014 (Carvalho et al. 2019). La legislación ambiental tiende a mirar hacia atrás en lugar de hacia adelante, enfatizando la protección de las condiciones históricas (que evita la pérdida de bosques primarios) en lugar de asegurar el futuro potencial de la restauración a escala de paisaje y la conectividad forestal, que influyen de forma clave en los niveles de biodiversidad y servicios ecosistémicos del futuro.

Otro aspecto a considerar es que los bosques regenerados naturalmente no están mapeados para fines de planificación y toma de decisiones, y cualquier ganancia en cobertura forestal rara vez se desagrega en sus componentes de bosque natural y plantado. Los cálculos de deforestación en la Amazonía de Brasil, utilizando el sistema nacional brasileño de monitoreo de deforestación por satélite PRODES, no incluyen la deforestación de bosques secundarios. Aun así, la tala de bosques secundarios y bosques para pasturas agroindustriales, plantaciones y actividades agrícolas a pequeña escala contribuye significativamente a la pérdida de bosques en algunas áreas. Una excepción constituye el sistema de mapeo TerraClass de uso del suelo utilizado para clasificar bosques secundarios, pasturas con vegetación leñosa y regeneración con pasturas en Brasil (Almeida et al, 2016). Este enfoque evidenció que un 19,2\% de áreas previamente deforestadas en el estado de Mato Grosso experimentaron regeneración natural. Boillat et al (2017) proponen un cambio radical en la manera como la cobertura boscosa se mapea e identifica, para así incorporar aspectos sociales y de uso del recurso que ayuden a una mejor toma de decisiones, más allá de la típica clasificación binaria de "bosque" y "no bosque". Esto ayudaría a "visibilizar" los bosques regenerados naturalmente.

Una barrera común es que cuando los pequeños propietarios permiten la regeneración natural, a menudo las leyes impiden manejar el bosque secundario joven o cosechar selectivamente madera y productos no maderables. En México, una vez que la regeneración natural alcanza una etapa en la que se define legalmente como bosque, las restricciones de cosecha, la necesidad de elaborar planes de corta y los altos costos de transacción conllevan a la reducción de los beneficios económicos que reciben los pequeños agricultores (Román-Dañobeytia et al, 2014). La ley forestal en Bután estipula que los bosques plantados en propiedad privada y comunal no requieren autorización estatal para la cosecha. Pero los árboles y bosques establecidos naturalmente, ya sea en tierras públicas o privadas, son patrimonio forestal nacional y requieren un plan de manejo y autorización antes de su utilización (Sears et al, 2018); y, además, la madera extraída de los bosques naturales está sujeta a impuestos.

Las políticas sectoriales y jurisdiccionales también obstaculizan la regeneración natural. La planificación del uso del suelo en Perú se acoge al mandato del Ministerio del Ambiente, pero es el Ministerio de Agricultura y Riego quien gobierna el cambio del uso del suelo mediante la emisión de títulos y permisos. Como resultado, el Ministerio del Ambiente tiene poca influencia para apoyar la conservación de la regeneración natural, a pesar de ser la agencia responsable de implementar pagos por bonos de carbono e incentivos relacionados (Kowler et al, 2016). De hecho, las directivas conflictivas entre los sectores del gobierno en el contexto de quién gobierna las intervenciones de restauración forestal (que incluyen la regeneración natural) están bastante extendidas en la mayoría de los países de América Latina (Schweizer et al, 2020).

\section{Opciones de políticas e innovaciones para favorecer la regeneración natural del bosque}

La regeneración natural ocurre bajo condiciones biofísicas, socioeconómicas y culturales específicas. Sin embargo, en la mayoría de los casos, es la consecuencia no intencional de otros procesos como la migración del campo a la ciudad, los cambios en los precios de los productos agrícolas y las políticas de exportación, el abandono de la agricultura en topografía montañosa que impide la mecanización, y restricciones gubernamentales sobre el uso de tierras agrícolas en propiedad privada o común. La regeneración natural ocurre intencionalmente cuando las áreas previamente deforestadas se incorporan nuevamente a áreas protegidas administradas por el Estado o se compran tierras privadas parcialmente deforestadas con la intención de conservar y restaurar el bosque nativo (AlgeetAbarquero et al, 2015), o cuando las comunidades deciden promover la regeneración como una forma de gestión para obtener productos forestales (Levy-Tacher et al, 2019). En comparación con zonas templadas y boreales, los enfoques de gestión de bosques regenerados naturalmente en el trópico se encuentran menos desarrollados. Los cambios necesarios en las políticas podrían ser posibles ahora que existen modelos computacionales que permiten la identificación de áreas específicas donde la regeneración natural del bosque es factible, costo efectiva y beneficiosa tanto para el medioambiente como para los medios de vida (Molin et al, 2018), lo cual puede promover un cambio en las políticas. Una planificación integral del uso del suelo y la aplicación de enfoques de priorización 
espacial (Chazdon y Guariguata 2018) pueden ayudar a que los bosques nativos se regeneren y persistan sin poner en peligro otras actividades productivas. Sin embargo, las políticas y mecanismos para empoderar las soluciones holísticas, incluyendo la expansión de sistemas agroforestales y silvopastoriles, se encuentran poco desarrolladas (Kremen y Merenlender, 2018). Serán necesarios incentivos económicos y políticos, en la medida en que las economías y los mercados pasan de aquellos que impulsan una mayor degradación de los bosques nativos a la restauración y mejora de los mismos. El desarrollo posterior de estos enfoques específicos deberá ir acompañado de políticas innovadoras en múltiples niveles de gobernanza para restaurar los bosques aprovechando la acción de la naturaleza.

Fomentar una transición en el ámbito forestal, particularmente donde la regeneración natural se promueve, representa grandes retos políticos e institucionales (Sloan, 2015). Estos desafíos no son insuperables, pero requerirán más investigación e innovaciones en políticas y arreglos de gobernanza a varios niveles jurisdiccionales. Por ejemplo, enfoques institucionales y de políticas que apoyan la intensificación agrícola, la protección forestal y los pagos por servicios ambientales en Costa Rica contribuyeron a un proceso de transición forestal que condujo a beneficios ambientales en general (Jadin et al, 2016), incluyendo las plantaciones de especies nativas y la regeneración natural del bosque (Calvo-Alvarado et al, 2019). La intensificación sostenible de la actividad agrícola y la integración de usos (land sharing) son objetivos clave para promover la seguridad alimentaria y el bienestar de los pequeños propietarios en los paisajes rurales (Latawiec et al, 2018). Fomentar la regeneración natural de los bosques nativos no es una opción viable para la restauración forestal si estos cambios no brindan beneficios a los habitantes rurales y si los bosques están destinados a tener una vida corta. Aunque en muchas regiones, los jóvenes y los adultos abandonan las áreas rurales y su futura relación con la tierra y los bosques (Paudel et al, 2014), aún hay tiempo de cambiar estas tendencias y de promover el resurgimiento del campo basado en la gestión proactiva e integrada del suelo, y en la restauración a gran escala del paisaje, donde los bosques y las nuevas generaciones puedan prosperar juntos.

\section{Referencias}

Aide T M, Grau H R, Graesser J, Andrade-Nuñez M J, Aráoz E, Barros A P, Campos-Cerqueira M, Chacon-Moreno E, Francisco C C y Espinoza R. 2019. Woody vegetation dynamics in the tropical and subtropical Andes from 2001 to 2014: satellite image interpretation and expert validation. Global Change Biology, 25, 2112-2126.

Aguiar A P, Vieira I C, Assis T, Dalla-Nora E, Toledo P, Santos-Junior R, Batistella M, Coelho A, Savaget E, Nobre C A y Ometto J P. 2016. Land use change emission scenarios: anticipating a forest transition process in the Brazilian Amazon? Global Change Biology, 22, 1821-1840.

Algeet-Abarquero N, Sánchez-Azofeifa A, Bonatti J. y Marchamalo M. 2015. Land cover dynamics in Osa Region, Costa Rica: secondary forest is here to stay. Regional Environmental Change, 15, 1461-1472.

Almeida C A D, Coutinho A C, Esquerdo J C D M, Adami M, Venturieri A, Diniz C G, Dessay N, Durieux L y Gomes A R. 2016 High spatial resolution land use and land cover mapping of the Brazilian Legal Amazon in 2008 using Landsat-5/TM and MODIS data. Acta Amazonica, 46, 291-302.
Álvarez-Berríos N L, Redo D J, Aide T M, Clark M L y Grau R. 2013. Land change in the Greater Antilles between 2001 and 2010. Land, 2, 81-107.

Bastin J-F, Finegold Y, Garcia C, Mollicone D, Rezende M, Routh D, Zohner C M y Crowther T W 2019. The global tree restoration potential. Science, 365, 76-79.

Bebi P, Seidl R, Motta R, Fuhr M, Firm D, Krumm F, Conedera M, Ginzler C, Wohlgemuth T y Kulakowski D. 2017. Changes of forest cover and disturbance regimes in the mountain forests of the Alps. Forest Ecology and Management, 388, 43-56.

Biggs D. 2018. Clearing,"Wasting," and Regreening: An Environmental History of Bare Hills in Central Vietnam. The Journal of Asian Studies, 77, 1037-1058.

Boillat S, Scarpa F M, Robson J P, Gasparri I, Aide T M, Aguiar A P D, Anderson L O, Batistella M, Fonseca M G et al. 2017. Land system science in Latin America: challenges and perspectives. Current Opinion in Environmental Sustainability, 26, 37-46.

Bonnesoeur V, Locatelli B, Guariguata M R, Ochoa-Tocachi B F, Vanacker V, Mao Z, Stokes A y Mathez-Stiefel S-L. 2019. Impacts of forests and forestation on hydrological services in the Andes: A systematic review. Forest Ecology and Management, 433, 569-584.

Borda-Niño M, Meli P y Brancalion P H S. 2020. Drivers of tropical forest cover increase: A systematic review. Land Degradation \& Development, doi: 10.1002/ldr.3534

Byg A, Novo P, Dinato M, Moges A, Tefera T, Balana B, Ldeamanuel T y Black H. 2017. Trees, soils, and warthogs-Distribution of services and disservices from reforestation areas in southern Ethiopia. Forest Policy and Economics, 84, 112-119.

Calaboni A, Tambosi L, Igari A, Farinaci J, Metzger JP y Uriarte M. 2018. The forest transition in São Paulo, Brazil: historical patterns and potential drivers. Ecology and Society, 23.

Calvo-Alvarado J, Jiménez V, Calvo-Obando A y Castillo M, 2019. Current perspectives on forest recovery trends in Guanacaste, Costa Rica. International Forestry Review, 21, 425-431.

Calvo-Alvarado J, McLennan B, Sanchez-Azofeifa A y Garvin T. 2009. Deforestation and forest restoration in Guanacaste, Costa Rica: Putting conservation policies in context. Forest Ecology and Management, 258, 931-940.

Carvalho R, Adami M, Amaral S, Bezerra F G y De Aguiar A P D. 2019. Changes in secondary vegetation dynamics in a context of decreasing deforestation rates in Pará, Brazilian Amazon. Applied Geography 106, 40-49.

Chazdon R L y Brancalion P H S. 2019. Restoring forests as a means to many ends. Science, 365, 24-25.

Chazdon R L y Guariguata M R. 2016. Natural regeneration as a tool for large-scale forest restoration in the tropics: prospects and challenges Biotropica, 48, 716-730.

Chazdon R L y Guariguata M R. 2018. Herramientas de apoyo a decisiones para la restauración del paisaje forestal: estado actual y futuro. Documentos Ocasionales 189. Bogor, Indonesia: CIFOR

Cochard R, Ngo D T, Waeber P O y Kull C A. 2017. Extent and causes of forest cover changes in Vietnam's provinces 1993-2013: A review and analysis of official data. Environmental Reviews, 25, 199-217.

Crouzeilles R, Barros F S, Molin PG, Ferreira M S, Junqueira AB, Chazdon R L, Lindenmayer D B, Tymus J R C, Strassburg B B N y Brancalion P H S. 2019. A new approach to map landscape variation in forest restoration success at the global scale. Journal of Applied Ecology, 56, 2675-2686

Crouzeilles R, Beyer HL, Monteiro L M, Feltran-Barbieri R, Pessôa ACM, Barros FSM, Lindenmayer D B, Lino EDS M, Grelle CEV, Chazdon R L, Matsumoto M, Rosa M, Latawiec A E y Strassburg BBN. 2020. Achieving cost-effective landscape-scale forest restoration through targeted natural regeneration. Conservation Letters, in press, DOl: 10.1111/conl.12709. 
Crouzeilles R, Ferreira M S, Chazdon R L, Lindenmayer D B, Sansevero J B B, Monteiro L, Iribarrem A, Latawiec A E y Strassburg B B N. 2017. Ecological restoration success is higher for natural regeneration than for active restoration in tropical forests. Science Advances, 3, e1701345.

Cruz-Alonso V, Ruiz-Benito P, Villar-Salvador P y Rey-Benayas J M. 2019. Long-term recovery of multifunctionality in Mediterranean forests depends onrestoration strategy and forest type. Journal of Applied Ecology, 56, 745-757.

da Silva R F B, Batistella M, Moran E F y Lu D. 2017. Land changes fostering Atlantic Forest transition in Brazil: Evidence from the Paraíba Valley. The Professional Geographer, 69, 80-93.

Díaz S, Settele J, Brondizio E S, Ngo H T, Guèze M, Agard J, Arneth A, Balvanera P, Brauman K, Butchart S et al. 2019. Summary for policymakers of the global assessment report of the Intergovernmental Science-Policy Platform on Biodiversity and Ecosystem Services. UN-IPBES, p. 39.

Ding H, Altamirano J C, Anchondo A, Faruqi S, Verdone M, Wu A, Zamora R, Chazdon R y Vergara W. 2017. Roots of prosperity: The economics and finance of restoring land. Washington, D. C.: Instituto de Recursos Mundiales.

Duangjai W, Schmidt-Vogt D y Shrestha R P. 2015. Farmers' land use decision-making in the context of changing land and conservation policies: A case study of Doi Mae Salong in Chiang Rai Province, Northern Thailand. Land Use Policy, 48, 179-189.

FAO 2015. State of Europe's Forests 2015. Ministerial Conference on the Protection of Forests in Europe, Forest Europe Liaison Unit, Madrid.

FAO y Global Mechanism of The UNCCD. 2015. Sustainable financing for forest and landscape restoration: Opportunities, challenges and the way forward. Discussion paper, Roma, FAO y UNCCD.

Filoso S, Bezerra M O, Weiss K C y Palmer M A. 2017. Impacts of forest restoration on water yield: A systematic review. PloS one, 12, e0183210

García-Barrios L, Galvan-Miyoshi Y M, Valdivieso-Perez I A, Masera, O R, Bocco G y Vandermeer J. 2009. Neotropical Forest Conservation, Agricultural Intensification, and Rural Out-migration: The Mexican Experience. Bioscience, 59, 863-873.

Gardner C J, Bicknell J E, Baldwin-Cantello W, Streubig M J y Davies Z G. 2019. Quantifying the impacts of defaunation on natural forest regeneration in a global meta-analysis. Nature Communications, 10,4590

Geddes L S, Lunt I D, Smallbone L T y Morgan J W. 2011. Old field colonization by native trees and shrubs following land use change: could this be Victoria's largest example of landscape recovery? Ecological Management \& Restoration, 12, 31-36.

Hecht S, Yang A L, Basnett BS, Padoch C y Peluso N L. 2015. People in motion, forests in transition: Trends in migration, urbanization, and remittances and their effects on tropical forests, CIFOR.

Jadin I, Meyfroidt P y Lambin E. 2016. International trade, and land use intensification and spatial reorganization explain Costa Rica's forest transition. Environmental Research Letters, 11, 035005.

Jakovac C C, Bongers F, Kuyper T W, Mesquita R C y Peña-Claros. 2016. Land use as a filter for species composition in Amazonian secondary forests. Journal of Vegetation Science, 27, 1104-1116.

Kowler L F, Ravikumar A, Larson A M, Rodriguez-Ward D, Burga C y Gonzales Tovar J. 2016. Analyzing multilevel governance in Peru. Working Paper 203. Bogor, Indonesia: CIFOR.

Kremen C y Merenlender A M. 2018. Landscapes that work for biodiversity and people. Science, 362, eaaau6020.

Kuemmerle T, Olofsson P, Chaskovskyy O, Baumann M, Ostapowicz K, Woodcock C E, Houghton R A, Hostert P, Keeton WS y Radeloff VC. 2011. Post-Soviet farmland abandonment, forest recovery, and carbon sequestration in western Ukraine. Global Change Biology, 17, 1335-1349.
Lacombe G, Ribolzi O, De Rouw A, Pierret A, Latsachak K, Silvera N, Pham Dinh R, Orange D, Janeau J y Soulileuth B. 2015.

Afforestation by natural regeneration or by tree planting: examples of opposite hydrological impacts evidenced by long-term field monitoring in the humid tropics. Hydrol. Earth Syst. Sci. Discuss, 12, 12615-12648.

Lambin E F y Meyfroidt P. 2010. Land use transitions: Socio-ecological feedback versus socio-economic change. Land Use Policy, 27, 108-118.

Lasanta T, Arnáez J, Pascual N, Ruiz-Flaño P, Errea M P y Lana-Renault N. 2017. Space-time process and drivers of land abandonment in Europe. Catena, 149, 810-823.

Latawiec A E, Dos Santos J S, Maioli V, Junqueira A B, Crouzeilles R, Jakovac C C, Tubenchlak F y Strassburg B B N. 2018. Forest landscape restoration and land sparing-sharing: shifting the focus towards nature's contributions to people, in: Mansourian, S, Parrotta, J. (Eds.), Forest Landscape Restoration. Routledge, London, pp. 116-134.

Lembani R L, Knight J y Adam E. 2019. Use of Landsat multi-temporal imagery to assess secondary growth Miombo woodlands in Luanshya, Zambia. Southern Forests, 81, 129-140.

Levy-Tacher S I, Ramírez-Marcial N, Navarrete-Gutiérrez D A y Rodríguez-Sánchez P V. 2019. Are Mayan community forest reserves effective in fulfilling people's needs and preserving tree species? Journal of Environmental Management, 245, 16-27.

Lindenmayer D B, Michael D, Crane M, Okada S, Barton P, Ikin Ky Florance D. 2016. Wildlife conservation in farm landscapes, CSIRO Publishing.

Lozano-Baez, S, Cooper, M, Frosini De Barros Ferraz, S, Ribeiro Rodrigues, R, Castellini, M y Di Prima S. 2019. Recovery of Soil Hydraulic Properties for Assisted Passive and Active Restoration: Assessing Historical Land Use and Forest Structure. Water, 11, 86.

Molin P G, Chazdon R L, Ferraz S F y Brancalion P H S. 2018. A landscape approach for cost-effectiven large-scale forest restoration. Journal of Applied Ecology, 55, 2767-2778.

Nanni A S, Sloan S, Aide T M, Graesser J, Edwards D y Grau H R. 2019. The neotropical reforestation hotspots: A biophysical and socioeconomic typology of contemporary forest expansion. Global Environmental Change, 54, 148-159.

Oldekop J A, Sims K R, Whittingham MJ y Agrawal A. 2018. An upside to globalization: International outmigration drives reforestation in Nepal. Global Environmental Change, 52, 66-74.

Paudel K P, Tamang S y Shrestha K K. 2014. Transforming land and livelihood: Analysis of agricultural land abandonment in the Mid Hills of Nepal. Journal of Forest and Livelihood, 12, 11-19.

Paudyal K, Baral H, Putzel L, Bhandari S y Keenan R. 2017. Change in land use and ecosystem services delivery from community-based forest landscape restoration in the Phewa Lake watershed, Nepal. International Forestry Review 19, 1-14.

Peltier R, Dubiez É, Diowo S, Gigaud M, Marien J N, Marquant B, Peroches A, Proces P y Vermeulen C. 2014. Assisted natural regeneration in slash-and-burn agriculture: Results in the democratic republic of the congo. Bois et Forets des Tropiques, 68 , 67-79.

Porras I y Chacón-Cascante A. 2018. Costa Rica's Payments for Ecosystem Services programme. Case study Module 2. In: PORRAS, I. y ASQUITH, N. (eds.) Ecosystems, poverty alleviation and conditional transfers. London: International Institute for Environment and Development.

Potapov P V, Turubanova S, Tyukavina A, Krylov A, Mccarty J, Radeloff $\checkmark$ y Hansen M. 2015. Eastern Europe's forest cover dynamics from 1985 to 2012 quantified from the full Landsat archive. Remote Sensing of Environment, 159, 28-43. 
Pugh T A, Lindeskog M, Smith B, Poulter B, Arneth A, Haverd V y Calle L. 2019. Role of forest regrowth in global carbon sink dynamics. Proceedings of the National Academy of Sciences, 116, 4382-4387.

Queiroz C, Beilin R, Folke C y Lindborg R. 2014. Farmland abandonment: threat or opportunity for biodiversity conservation? A global review. Frontiers in Ecology and the Environment, 12, 288-296.

Reid J L, Fagan M E, Lucas J, Slaughter J y Zahawi R A. 2019. The ephemerality of secondary forests in southern Costa Rica. Conservation Letters, 12, e12607.

Rey Benayas J M, Bullock J M y Newton A C. 2008. Creating woodland islets to reconcile ecological restoration, conservation, and agricultural land use. Frontiers in Ecology and the Environment, 6, 329-336.

Román-Dañobeytia F J, Levy-Tacher S I, Macario-Mendoza P y Zúñiga-Morales J. 2014. Redefining secondary forests in the Mexican Forest Code: Implications for management, restoration and conservation. Forests, 5, 978-991.

Rozendaal D M A, Bongers F, Aide T M, Alvarez-Dávila E, Ascarrunz, N, Balvanera, P, Becknell J M, Bentos TV, Brancalion P H S, Cabral G A $L$ et al. 2019. Biodiversity recovery of Neotropical secondary forests. Science Advances, 5, eaau3114.

Rudel T K, Sloan S, Chazdon R y Grau R. 2016. The drivers of tree cover expansion: Global, temperate, and tropical zone analyses. Land Use Policy, 58, 502-513.

Schwartz N B, Uriarte M, Defries R, Gutierrez-Velez V y PinedoVasquez M. 2017. Land-use dynamics influence estimates of carbon sequestration potential in tropical second-growth forest. Environmental Research Letters, 12, 074023.

Schweizer D, Meli P, Brancalion P H S y Guariguata M R. 2020. Implementing forest landscape restoration in Latin America: Stakeholder perceptions on legal frameworks. Land Use Policy, https://doi.org/10.1016/j.landusepol.2019.104244.

San Roman Sanz A, Fernandez C, Mouillot F, Ferrat L, Istria D y Pasqualini V. 2013. Long-term forest dynamics and land-use abandonment in the Mediterranean Mountains, Corsica, France. Ecology and Society, 18, 38.

Sato C F, Wood J T, Stein J A, Crane M, Okada S, Michael D R, Kay, G M, Florance D, Seddon J y Gibbons P. 2016. Natural tree regeneration in agricultural landscapes: The implications of intensification. Agriculture, Ecosystems \& Environment, 230, 98-104.
Sears R, Choden K, Dorji T, Dukpa D, Phuntsho S, Rai P, Wangchuk J y Baral H. 2018. Bhutan's Forests through the Framework of Ecosystem Services: Rapid Assessment in Three Forest Types. Forests, 9, 675

Shaver I, Chain-Guadarrama A, Cleary K A, Sanfiorenzo A, SantiagoGarcía R J, Finegan B, Hormel L, Sibelet N, Vierling L A y BosquePérez N A. 2015. Coupled social and ecological outcomes of agricultural intensification in Costa Rica and the future of biodiversity conservation in tropical agricultural regions. Global Environmental Change, 32, 74-86.

Sloan S. 2015. The development-driven forest transition and its utility for REDD+. Ecological Economics, 116, 1-11.

Sloan S, Goosem M y Laurance S G. 2016. Tropical forest regeneration following land abandonment is driven by primary rainforest distribution in an old pastoral region. Landscape Ecology, 31 601-618.

Smale M, Tappan G y Reij C. 2018. Chapter 1. Farmer-managed restoration of agroforestry parklands in Niger. Fostering transformation and growth in Niger's agricultural sector. Wageningen Academic Publishers

Song X-P, Hansen M C, Stehman S V, Potapov P V, Tyukavina A,Vermote E F y Townshend J R. 2018. Global land change from 1982 to 2016. Nature, 560, 639

Strassburg BN, Barros F S M, Couzeilles R, Iribarrem A, Santos JS, Silva D, Sansevero JBB, Alves-Pinto H, Feltran-Barbieri R y Latawiec A. 2016. The role of natural regeneration to ecosystem services provision and habitat availability: a case study in the Brazilian Atlantic Forest. Biotropica, 48, 890-899.

Uriarte M y Chazdon R L. 2016. Incorporating natural regeneration in forest landscape restoration in tropical regions: Synthesis and key research gaps. Biotropica, 48, 915-924

Wang C, Yu M y Gao Q. 2017. Continued reforestation and urban expansion in the new century of a tropical island in the Caribbean. Remote Sensing, 9, 731

Yang Y, Wang L, Yang Z, Xu C, Xie J, Chen G, Lin C, Guo J, Liu X y Xiong D. 2018. Large ecosystem service benefits of assisted natural regeneration. Journal of Geophysical Research: Biogeosciences, 123, 676-687.

Zahawi R A, Reid J L y Holl K D. 2014. Hidden costs of passive restoration. Restoration Ecology, 22, 284-287.

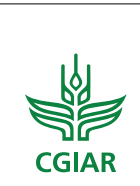

Esta investigación fue realizada por CIFOR como parte del Programa de Investigación de CGIAR sobre Bosques, Árboles y Agroforestería (FTA). El FTA es el programa de investigación para el desarrollo más grande del mundo, dedicado a mejorar el papel de bosques, árboles y la agroforestería para el desarrollo sostenible, la seguridad alimentaria, y frente al cambio climático. CIFOR dirige el programa FTA en asociación con Bioversity International, CATIE, CIRAD, ICRAF, INBAR y TBI.

La investigación del Programa FTA cuenta con el apoyo del Fondo Fiduciario del CGIAR: cgiar.org/funders

\section{USAID}

